\title{
POSTER
}

\section{Prise en charge des fractures de l'angle mandibulaire : illustration par deux cas cliniques.}

\section{Muret $\mathbf{M}^{1}$, De Boutray $\mathrm{M}^{1}$, Torres $\mathrm{JH}^{2}$, Fauroux $\mathrm{MA}^{2}$}

1. Chirurgie Maxillo-Faciale Stomatologie et Chirurgie Plastique et Reconstructrice de la face - $\mathrm{CHU}$ Montpellier

2. Chirurgie Orale - Centre de Soins d'Enseignement et de Recherche Dentaires - CHU Montpellier

\section{Introduction}

Après les fractures isolées des os nasaux, les fractures de mandibule sont les plus fréquentes de la face. Elles concernent principalement l'adulte jeune de sexe masculin. En France, elles sont d'abord dues aux rixes, puis aux accidents de la voie publique (1). Leur prise en charge, chirurgicale ou non, dépend du nombre et du déplacement des foyers fracturaires, de l'orientation de la fracture, du type de fracture, de la présence de complications, du terrain. Dans tous les cas, un traitement dentaire (au minimum un protocole d'hygiène) et des règles diététiques (2) sont nécessaires.

\section{Observations}

Le premier cas proposé est celui d'un homme âgé de vingt ans victime d'une agression. Quelques heures après, il a présenté une douleur mandibulaire spontanée, un trismus, une tuméfaction jugale en regard de l'angle mandibulaire droit et un trouble de l'articulé dentaire. Le scanner montrait une fracture bicorticale de l'angle droit, avec refend sur la branche définissant un troisième fragment. Un traitement chirurgical a été pratiqué : après réduction et blocage peropératoire sur fils d'acier, les foyers de fracture ont été ostéosynthésés. En post-opératoire immédiat, un contrôle radiologique a été réalisé. Les règles diététiques ont été dispensées : trois périodes de quinze jours pendant lesquelles se sont succédées une alimentation liquide puis mixée et enfin molle. Un protocole d'hygiène buccodentaire a été instauré avec une brosse à dents chirurgicale et des bains de bouche sans alcool. Des séances de kinésithérapie ont été prescrites. L'évolution a été contrôlée au quinzième jour.

Le second cas est celui d'un homme âgé de trente-neuf ans ayant chuté de vélo. La fracture est passée inaperçue le jour de l'accident. Mais, devant la persistance de la gêne masticatoire, le patient a consulté au septième jour. L'examen clinique a mis en évidence une douleur mandibulaire à la palpation et à la mastication d'une alimentation solide, une absence de trismus, un articulé dentaire conservé. Une fracture unifocale bicorticale complète non déplacée de l'angle mandibulaire droit a été constatée à l'orthopantomogramme. L'intervention chirurgicale n'a pas été retenue puisqu'il s'agissait d'une fracture unifocale sans déplacement ni comorbidités, chez un patient compliant. Les règles hygiéno-diététiques lui ont été proposées. Pendant six semaines, une évolution hebdomadaire a été suivie à l'aide de contrôle radiologique.

\section{Discussion}

Ces deux cas s'opposent par leur symptomatologie. La mobilité des fragments et le déplacement des foyers fracturaires sont les principaux critères évalués pour déterminer la stabilité de la fracture. En conséquence, l'indication est ou non chirurgicale.

\section{Conclusion}

Les cas rapportés illustrent le fondement de la prise en charge chirurgicale : la stabilité de la fracture.

m-muret@chu-montpellier.fr

\section{Références}

(1) Rocton S et al. Rev. Stomatol. Chir. Maxillofac. 2007 ; 108 : 3-12.

(2) Mercier J et al. Rev. Stomatol. Chir. Maxillofac. $2000 ; 101$ : 203-206.

Autorisation de changement de catégorie à la Commission Scientifique et de la Recherche de la SFCO.

(C) The authors, published by EDP Sciences. This is an Open Access article distributed under the terms of the Creative Commons Attribution License 4.0 (http://creativecommons.org/licenses/by/4.0/) 